\title{
COMPLETELY INTEGRABLE TORUS ACTIONS ON COMPLEX MANIFOLDS WITH FIXED POINTS
}

\author{
HIROAKI ISHIDA AND YAEL KARSHON
}

\begin{abstract}
We show that if a holomorphic $n$-dimensional compact torus action on a compact connected complex manifold of complex dimension $n$ has a fixed point then the manifold is equivariantly biholomorphic to a smooth toric variety.
\end{abstract}

\section{Introduction}

We begin by recalling some notions from the theory of toric varieties.

We work in the vector space $\operatorname{Lie}\left(S^{1}\right)^{n} \cong \mathbb{R}^{n}$ with the lattice $\operatorname{Hom}\left(S^{1},\left(S^{1}\right)^{n}\right) \cong \mathbb{Z}^{n}$. Here, we identify $\operatorname{Lie}\left(S^{1}\right)$ with $\mathbb{R}$ such that the exponential map exp: $\mathbb{R} \rightarrow S^{1}$ is $t \mapsto e^{2 \pi i t}$

A unimodular fan is a finite set $\Delta$ of convex polyhedral cones with the following properties.

(1) A face of a cone in $\Delta$ is also a cone in $\Delta$.

(2) The intersection of two cones in $\Delta$ is a common face.

(3) Every cone in $\Delta$ is unimodular, i.e., it has the form $\operatorname{pos}\left(\lambda_{1}, \ldots, \lambda_{k}\right)$ where $\lambda_{1}, \ldots, \lambda_{k}$ is part of a $\mathbb{Z}$-basis of the lattice. Here, pos denotes the positive span: the set of linear combinations with non-negative coefficients. ${ }^{1}$

A fan $\Delta$ is complete if the union of the cones in $\Delta$ is all of $\operatorname{Lie}\left(S^{1}\right)^{n}$.

The theory of toric varieties associates to a unimodular fan $\Delta$ a complex manifold $M_{\Delta}$ with a holomorphic $\left(\mathbb{C}^{*}\right)^{n}$-action with the following properties.

(1) The fixed points in $M_{\Delta}$ are in bijection with the $n$-dimensional cones in $\Delta$.

(2) Let $p$ be a fixed point in $M_{\Delta}$. Then the isotropy weights at $p$ are a $\mathbb{Z}$-basis to the lattice $\operatorname{Hom}\left(\left(S^{1}\right)^{n}, S^{1}\right) \subset\left(\operatorname{Lie}\left(S^{1}\right)^{n}\right)^{*}$. Moreover, let $\lambda_{1}, \ldots, \lambda_{n}$ be the dual basis; then the cone in $\Delta$ that corresponds to $p$ is $\operatorname{pos}\left(\lambda_{1}, \ldots, \lambda_{n}\right)$.

(3) The manifold $M_{\Delta}$ is compact if and only if the fan $\Delta$ is complete.

Explicitly, let $\lambda_{1}, \ldots, \lambda_{m} \in \mathbb{Z}^{n}$ be the primitive generators of the one-dimensional cones in $\Delta$. Each $\lambda_{i}$ encodes a homomorphism $a \mapsto a^{\lambda_{i}}$ from $\mathbb{C}^{*}$ to $\left(\mathbb{C}^{*}\right)^{n}$; together they give a homomorphism $\pi:\left(a_{1}, \ldots, a_{m}\right) \mapsto \prod_{j=1}^{m} a_{j}^{\lambda_{j}}$ from $\left(\mathbb{C}^{*}\right)^{m}$ to $\left(\mathbb{C}^{*}\right)^{n}$. Then $M_{\Delta}=U_{\Delta} / K_{\Delta}$, where $U_{\Delta}=\left\{z \in \mathbb{C}^{m} \mid \operatorname{pos}\left(\lambda_{i} \mid z_{i}=0\right) \in \Delta\right\}$ and $K_{\Delta}=\operatorname{ker} \pi$. For the details of the construction and the proof of its properties, we refer the reader to the book [3] by Cox et al. and to the book [1] by Audin.

Received by the editors March 23, 2012.

2010 Mathematics Subject Classification. Primary 14M25, Secondary 32M05, 57S25.

Key words and phrases. Torus action, complex manifold, toric manifold.

${ }^{1}$ This property of a cone or a fan is also described in the literature by the adjectives smooth, non-singular, regular, and Delzant. 
In fact, $M_{\Delta}$ is an algebraic variety. Moreover, every smooth complex algebraic variety that is equipped with an algebraic $\left(\mathbb{C}^{*}\right)^{n}$-action with an open dense free orbit is isomorphic to some $M_{\Delta}$. (The proof of this fact appeared in the book [11] by Kempf et al. and in the paper [15] by Miyake and Oda and relies on a lemma of Sumihiro [16]; see Corollary 3.1.8 in [3].) Our main theorem is a complex analytic variant of this result:

Theorem 1. Let $M$ be a connected complex manifold of complex dimension $n$ equipped with a faithful action of the torus $\left(S^{1}\right)^{n}$ by biholomorphisms. If $M$ is compact and the action has fixed points, then there exists a unimodular fan $\Delta$ and an $\left(S^{1}\right)^{n}$-equivariant biholomorphism of $M_{\Delta}$ with $M$.

\section{Remark 2.}

(1) Our theorem gives a negative answer to a question that was raised by Buchstaber and Panov in [2, Problem 5.23].

Let $M$ be a closed $2 n$-dimensional manifold with an $\left(S^{1}\right)^{n}$-action that is locally standard: every orbit has a neighbourhood that is equivariantly diffeomorphic, up to an automorphism of $\left(S^{1}\right)^{n}$, to an invariant open subset of $\mathbb{C}^{n}$ with the standard $\left(S^{1}\right)^{n}$-action. Also assume that the quotient $M /\left(S^{1}\right)^{n}$ is diffeomorphic, as a manifold with corners, to a simple convex polytope $P$ in $\mathbb{R}^{n}$. $^{2}$ Such manifolds, introduced in [4] and studied in the toric topology community, are called quasi-toric manifolds ${ }^{3}$.

The question of Buchstaber and Panov is whether there exists a non-toric quasitoric manifold that admits an $\left(S^{1}\right)^{n}$-invariant complex structure.

(2) Our theorem strengthens an earlier result of Ishida and Masuda, that if a closed complex manifold of complex dimension $n$ admits an $\left(S^{1}\right)^{n}$-action, and if its odd-degree cohomology groups vanish, then the Todd genus of the manifold is equal to one. See [9, Theorem 1.1 and Remark 1.2].

(3) In Theorem 1, the assumption "complex" cannot be weakened to "almost complex". For example, for every two complex toric manifolds of complex dimension 2 , their equivariant connected sum along a free orbit supports an invariant almost complex structure, has fixed points, but is not (equivariantly diffeomorphic to) a toric manifold; see [10, Section 11.2]. For higher-dimensional analogues, see [6, Section 13]; for more interesting four-dimensional examples, see [14, Theorem 5.1]. A necessary and sufficient condition for a quasitoric manifold to admit an invariant almost complex structure was given in $[13$, Theorem 1].

(4) The symplectic analogue of Theorem 1 is also true: a closed symplectic manifold of dimension $2 n$ with a faithful $\left(S^{1}\right)^{n}$ action with at least one fixed point is a symplectic toric manifold. To see this, it is enough to show that such an action is Hamiltonian; being a toric manifold then follows from Delzant's

\footnotetext{
${ }^{2}$ A map from $M /\left(S^{1}\right)^{n}$ to $P$ is a diffeomorphism of manifolds with corners if and only if it is a homeomorphism and, for every real valued function on $P$, the function extends to a smooth function on $\mathbb{R}^{n}$ if and only if its pullback to $M$ is smooth. For every $k \in\{0, \ldots, n\}$, a diffeomorphism carries the $k$-dimensional orbits in $M$ to the relative interiors of the $k$-dimensional faces of $P$.

${ }^{3}$ Davis-Januszkiewicz [4] used the term toric manifold, but this term was already used in the literature to mean a smooth toric variety, so Buchstaber-Panov [2] introduced instead the term quasitoric manifold.
} 
theorem [5, Théorème 2.1]. Let $p$ a fixed point. There exist $n$ subcircles of $\left(S^{1}\right)^{n}$ that span $\left(S^{1}\right)^{n}$ and whose isotropy weights are all positive. In order to show that the $\left(S^{1}\right)^{n}$ action is Hamiltonian, it is enough to show that each of these $S^{1}$ actions has a momentum map. Fix one of these $S^{1}$ actions. Since there is a fixed point, the $S^{1}$ orbits are null-homotopic, so the $S^{1}$ action lifts to an $S^{1}$ action on the universal bundle, $\tilde{M}$. As $H^{1}(\tilde{M})=0$, this lifted action is Hamiltonian. By Morse theory, at most one point of $\tilde{M}$ can be a strict local minimum for the momentum map (see, e.g., [7]). So the fibre of $\tilde{M}$ over the fixed point $p$ can contain only one point. So $\tilde{M}=M$, and so there is a momentum map on M.

(5) It is necessary to assume that the action has fixed points: the complex torus $\mathbb{C}^{*} /(z \sim 2 z)$ has a holomorphic $S^{1}$-action, induced from multiplication on $\mathbb{C}^{*}$, but it is not a toric variety: the $\mathbb{C}^{*}$-action is not faithful.

(6) It is necessary to assume that the manifold is compact: the open unit disc in $\mathbb{C}$ with the natural circle action has a fixed point, but it is not a toric variety: the circle action does not extend to a $\mathbb{C}^{*}$-action.

\section{The complexified action}

Let the torus $\left(S^{1}\right)^{n}$ act on a complex manifold $M$ by biholomorphisms. If the manifold $M$ is compact, then the $\left(S^{1}\right)^{n}$-action extends to a $\left(\mathbb{C}^{*}\right)^{n}$-action that is holomorphic not only in the sense that each element of $\left(\mathbb{C}^{*}\right)^{n}$ acts by a biholomorphism but also in the sense that the action map $\left(\mathbb{C}^{*}\right)^{n} \times M \rightarrow M$ is holomorphic. See, e.g., [8, Theorem 4.4]. For the convenience of the reader, we briefly recall here some of the details of this standard construction.

Let $\xi_{1}, \ldots, \xi_{n}$ be the fundamental vector fields of the $\left(S^{1}\right)^{n}$-action with respect to the coordinate one-dimensional subtori. Let $J: T M \rightarrow T M$ be the multiplication by $\sqrt{-1}$. We claim that the vector fields $-J \xi_{1}, \ldots,-J \xi_{n}$ are holomorphic (in the sense that their flows preserve the complex structure) and commute with each other and with the vector fields $\xi_{i}$.

As the $\left(S^{1}\right)^{n}$-action preserves $J$ and $\xi_{j}$, it preserves $-J \xi_{j}$, for each $j$. So the vector fields $-J \xi_{j}$ commute with the vector fields $\xi_{i}$ that generate this action. Since $J$ is a complex structure, its Nijenhuis tensor,

$$
N(Z, W):=2([J Z, J W]-J[Z, J W]-J[J Z, W]-[Z, W]),
$$

vanishes. Setting $Z=\xi_{i}$ and $W=\xi_{j}$, we get that $\left[J \xi_{i}, J \xi_{j}\right]=J\left[\xi_{i}, J \xi_{j}\right]+J\left[J \xi_{i}, \xi_{j}\right]+$ $\left[\xi_{i}, \xi_{j}\right]$, and each of the three terms on the right hand side is zero. So the vector fields $-J \xi_{j}$ commute with each other. A vector field $Y$ is holomorphic if and only if $[Y, J W]=J[Y, W]$ for each vector $W$; see [12, Proposition 2.10 in Chapter IX]. Set $Y:=-J \xi_{i}$ and $W$ arbitrary; because $J Y\left(=\xi_{i}\right)$ is holomorphic, $[J Y, J W]=J[J Y, W]$; by the vanishing of the Nijenhuis tensor,

$$
\begin{aligned}
0=N(J Y, W) & =2([-Y, J W]-J[J Y, J W]-J[-Y, W]-[J Y, W]) \\
& =2([-Y, J W]-J[-Y, W]),
\end{aligned}
$$

so $Y$ is holomorphic. 
If $M$ is compact, the vector fields $-J \xi_{1}, \ldots,-J \xi_{n}$ are complete, and we get an $\mathbb{R}^{2 n}$-action, $\mathbb{R}^{2 n} \times M \rightarrow M$, via

$$
\left(\sum_{i=1}^{2 n} a_{i} \mathbf{e}_{i}, x\right) \mapsto c_{x}(1),
$$

where $c_{x}(r)$ is the integral curve of the vector field $\sum_{i=1}^{n}-a_{i} J \xi_{i}+a_{n+i} \xi_{i}$ with $c_{x}(0)=$ $x$. This action descends to a $\left(\mathbb{C}^{*}\right)^{n}$-action by biholomorphisms that extends the given $\left(S^{1}\right)^{n}$-action. Finally, the action map $\left(\mathbb{C}^{*}\right)^{n} \times M \rightarrow M$ is holomorphic, because its differential, which at the point $(z, m)$ is the map $\mathbb{C}^{n} \times T_{m} M \rightarrow T_{z \cdot m} M$ that takes $\left(2 \pi\left(r_{1}+i \theta_{1}, \ldots, r_{n}+i \theta_{n}\right), v\right)$ to $\sum_{j}-\left.r_{j} J \xi_{j}\right|_{z \cdot m}+\left.\theta_{j} \xi_{j}\right|_{z \cdot m}+z_{*} v$, is complex linear.

Remark 3. In the next section we will see that if there exists a fixed point then the extended $\left(\mathbb{C}^{*}\right)^{n}$-action is faithful. In general, the extended $\left(\mathbb{C}^{*}\right)^{n}$-action might not be faithful.

Example 4. Let $\left(S^{1}\right)^{n}$ act on $\mathbb{C}^{n}$ with weights $\alpha_{1}, \ldots, \alpha_{n}$ :

$$
g \cdot\left(z_{1}, \ldots, z_{n}\right)=\left(g^{\alpha_{1}} z_{1}, \ldots, g^{\alpha_{n}} z_{n}\right),
$$

where $g^{\alpha_{i}}=g_{1}^{\alpha_{i 1}} \cdots g_{n}^{\alpha_{i n}}$ for $g=\left(g_{1}, \ldots, g_{n}\right) \in\left(S^{1}\right)^{n}$ and for the isotropy weight $\alpha_{i}=\left(\alpha_{i 1}, \ldots, \alpha_{i n}\right) \in \mathbb{Z}^{n}$. Then the complexified action is given by the same formula applied to $g=\left(g_{1}, \ldots, g_{n}\right) \in\left(\mathbb{C}^{*}\right)^{n}$.

\section{Structures near fixed points}

Let $M$ be a connected complex manifold of complex dimension $n$. Let the torus $\left(S^{1}\right)^{n}$ act on $M$ faithfully by biholomorphisms. Let $p$ be a point in $M$ that is fixed by the $\left(S^{1}\right)^{n}$-action. Let $\alpha_{1}, \ldots, \alpha_{n}$ be the isotropy weights at $p$.

Let $\mathbb{C}_{\alpha_{i}}$ denote the one-dimensional complex vector space $\mathbb{C}$ with the $\left(S^{1}\right)^{n}$-action that is obtained by composing the homomorphism $\left(S^{1}\right)^{n} \rightarrow S^{1}$ that is encoded by the weight $\alpha_{i}$ with the standard action of $S^{1}$ on $\mathbb{C}$ by scalar multiplication.

We begin with a local result:

Lemma 5. There exists an $\left(S^{1}\right)^{n}$-invariant neighbourhood $U_{p}$ of $p$ in $M$, an $\left(S^{1}\right)^{n}$ invariant neighbourhood $\widetilde{U}_{p}$ of the origin in $T_{p} M$, and an $\left(S^{1}\right)^{n}$-equivariant biholomorphism $\varphi_{p}: U_{p} \rightarrow \widetilde{U}_{p}$ whose differential at $p$ is the identity map on $T_{p} M$.

Proof. Let $\varphi: U \rightarrow \widetilde{U} \subseteq \mathbb{C}^{n}$ be a local holomorphic chart near $p$ with $\varphi(p)=0$. Identifying $\mathbb{C}^{n}$ with $T_{p} M$ via the differential

$$
(d \varphi)_{p}: T_{p} M \rightarrow T_{0} \mathbb{C}^{n} \cong \mathbb{C}^{n}
$$

we get a biholomorphism

$$
\varphi^{\prime}: U \rightarrow \widetilde{U}^{\prime} \subseteq T_{p} M
$$

whose differential at $p$ is the identity map on $T_{p} M$. We want to obtain such a biholomorphism that is also equivariant.

Set

$$
U^{\prime}:=\bigcap_{g \in\left(S^{1}\right)^{n}} g U
$$


Clearly, $U^{\prime}$ is invariant and contains $p$. We now show that $U^{\prime}$ is open. The complement of $U^{\prime}$ is the image of the closed subset $\left(S^{1}\right)^{n} \times(M \backslash U)$ of $\left(S^{1}\right)^{n} \times M$ under the action map $\left(S^{1}\right)^{n} \times M \rightarrow M$. Since $\left(S^{1}\right)^{n}$ is compact, the action map is proper. Being proper means that the preimage of every compact set is compact; when the target space $M$ is a manifold ${ }^{4}$ it implies that the map is closed. Thus, the complement $M \backslash U^{\prime}$ is closed, and so $U^{\prime}$ is open.

To obtain an equivariant chart, we average $\varphi^{\prime}$ : let

$$
\widetilde{\varphi}:=\int_{g \in\left(S^{1}\right)^{n}}\left(g \circ \varphi^{\prime} \circ g^{-1}\right) d g: U^{\prime} \rightarrow T_{p} M
$$

where $d g$ is Haar measure on $\left(S^{1}\right)^{n}$. The map $\widetilde{\varphi}$ is holomorphic and $\left(S^{1}\right)^{n}$-equivariant. Moreover, its differential at $p$ is the identity map on $T_{p} M$. By the implicit function theorem, $\widetilde{\varphi}$ restricts to a biholomorphism from some smaller open neighbourhood $U^{\prime \prime}$ of $p$ in $M$ to an open neighbourhood of the origin in $T_{p} M$. The restriction of $\widetilde{\varphi}$ to the invariant neighbourhood $U_{p}:=\bigcap_{g \in\left(S^{1}\right)^{n}} g \cdot U^{\prime \prime}$ of $p$ in $M$ satisfies the requirements of the lemma.

Corollary 6. There exists an $\left(S^{1}\right)^{n}$-equivariant local holomorphic chart

$$
\varphi_{p}: U_{p} \rightarrow \mathbb{D}^{n}
$$

from an invariant open neighbourhood $U_{p}$ of $p$ to a polydisc $\mathbb{D}^{n}$ in $\mathbb{C}_{\alpha_{1}} \oplus \ldots \oplus \mathbb{C}_{\alpha_{n}}$.

Proof. By the definition of the isotropy weights, there exists a complex linear $\left(S^{1}\right)^{n}$ equivariant isomorphism between the tangent space $T_{p} M$ and the representation $\mathbb{C}_{\alpha_{1}} \oplus$ $\ldots \oplus \mathbb{C}_{\alpha_{n}}$. Corollary 6 then follows from Lemma 5 by restricting the chart to the preimage of a polydisc.

We would like to extend the chart of Corollary 6 to a chart whose image is all of $\mathbb{C}^{n}$. We can do this when the $\left(S^{1}\right)^{n}$ extends to a $\left(\mathbb{C}^{*}\right)^{n}$-action; for example, if the manifold is compact; by "sweeping" by the $\left(\mathbb{C}^{*}\right)^{n}$-action.

Lemma 7. Suppose that the $\left(S^{1}\right)^{n}$-action extends to a $\left(\mathbb{C}^{*}\right)^{n}$-action. Then there exists an invariant open neighbourhood $V_{p}$ of $p$ in $M$ and an $\left(S^{1}\right)^{n}$-equivariant biholomorphism of $V_{p}$ with $\mathbb{C}_{\alpha_{1}} \oplus \cdots \oplus \mathbb{C}_{\alpha_{n}}$.

Proof. Let $\varphi_{p}: U_{p} \rightarrow \mathbb{D}^{n}$ be an $\left(S^{1}\right)^{n}$-equivariant holomorphic local chart, as in Corollary 6. Since $\varphi_{p}$ is $\left(S^{1}\right)^{n}$-equivariant and holomorphic, it intertwines the restriction to $U_{p}$ of the vector fields that generate the complexified $\left(\mathbb{C}^{*}\right)^{n}$-action on $M$ with the restriction to $\mathbb{D}^{n}$ of the vector fields that generate the complexified $\left(\mathbb{C}^{*}\right)^{n}$-action on $\mathbb{C}^{n}=\mathbb{C}_{\alpha_{1}} \oplus \cdots \oplus \mathbb{C}_{\alpha_{n}}$. This, and the fact that $\varphi_{p}$ is a diffeomorphism between $U_{p}$ and $\mathbb{D}^{n}$, implies that $\varphi_{p}$ also intertwines the partial flows on $U_{p}$ and on $\mathbb{D}^{n}$ that are generated by these vector fields; in particular it intertwines the domains of definition of these partial flows.

For each $t \in \mathbb{R}$, let $g_{t}$ be the element of $\left(\mathbb{C}^{*}\right)^{n}$ that acts on $\mathbb{C}^{n}$ as scalar multiplication by $e^{-t}$, and let $\eta \in \operatorname{Lie}\left(\mathbb{C}^{*}\right)^{n}$ be the generator of the one-parameter subgroup $t \mapsto g_{t}$. Since $e^{-t} \mathbb{D}^{n} \subset \mathbb{D}^{n}$ for all $t \geq 0$, and because $\varphi_{p}$ intertwines the domains

\footnotetext{
${ }^{4}$ In fact, it is enough to assume that the target space is Hausdorff and compactly generated. Compactly generated means that a subset is closed if and only if its intersection with every compact set $K$ is closed in $K$; this property holds if the space is locally compact or if the space is metrizable.
} 
of definition of the partial flows on $U_{p}$ and on $\mathbb{D}^{n}$ that correspond to $\eta$, we get that $g_{t} U_{p} \subset U_{p}$ for all $t \geq 0$. So, for every $t \geq 0$, the domain of definition of the $\left(S^{1}\right)^{n}$-equivariant biholomorphism

$$
\varphi_{p}^{(t)}:=\left(g_{t}\right)^{-1} \circ \varphi_{p} \circ g_{t}: g_{-t} U_{p} \rightarrow e^{t} \mathbb{D}^{n}
$$

contains $U_{p}$. Here, $g_{t}: g_{-t} U_{p} \rightarrow U_{p}$ and $\left(g_{t}\right)^{-1}: \mathbb{D}^{n} \rightarrow e^{t} \mathbb{D}^{n}$ are given by the complexified actions on $M$ and on $\mathbb{C}^{n}$. By the choice of $g_{t}$, the latter map is multiplication by $e^{t}$.

Moreover, because $\varphi_{p}$ intertwines the partial flows that correspond to $\eta$ and these partial flows are defined for all $t \geq 0$, the restriction to $U_{p}$ of $\varphi_{p}^{(t)}$ coincides with $\varphi_{p}$ for all $t \geq 0$. Substituting $t-s$ instead of $t$, we get that the maps $\varphi_{p}^{(t)}$ and $\varphi_{p}^{(s)}$ agree whenever they are both defined. Thus, all these maps fit together into a map

$$
\bigcup_{t \geq 0} \varphi_{p}^{(t)}: V_{p} \rightarrow \mathbb{C}_{\alpha_{1}} \oplus \cdots \oplus \mathbb{C}_{\alpha_{n}}
$$

where $V_{p}=\bigcup_{t \geq 0} g_{-t} U_{p}$. This map is onto, because its image is the union of the sets $e^{t} \mathbb{D}^{n}$ over all $t \geq 0$. The map is one to one, because it is one to one on each $g_{-t} U_{p}$, and for every two points in the domain there exists a $t \geq 0$ such that the points are both in $g_{-t} U_{p}$. Since $V_{p}$ is covered by $\left(S^{1}\right)^{n}$-invariant open sets $g_{-t} U_{p}$ on which the map is an $\left(S^{1}\right)^{n}$-equivariant biholomorphism, we deduce that the map is itself an $\left(S^{1}\right)^{n}$-equivariant biholomorphism, as required.

\section{Obtaining a fan}

Let $M$ be a connected complex manifold of complex dimension $n$, let the torus $\left(S^{1}\right)^{n}$ act on $M$ faithfully by biholomorphisms, and assume that this action extends to a holomorphic $\left(\mathbb{C}^{*}\right)^{n}$-action. The set of fixed points is discrete; assume that it is nonempty and finite.

In Lemma 7 we assigned to every fixed point $p$ in $M$ an open subset $V_{p}$ that is biholomorphic to $\mathbb{C}^{n}$. By assumption, there exists at least one fixed point. So, the union $X$ of the sets $V_{p}$ over these fixed points,

$$
X:=\bigcup_{p \in M^{\left(S^{1}\right)^{n}}} V_{p},
$$

is nonempty.

Remark 8. In Section 6, we show that if $M$ is compact and connected then the union $X$ of the sets $V_{p}$ is all of $M$. The proof relies on the results of Sections 4 and 5 .

By its definition, $X$ is a $\left(\mathbb{C}^{*}\right)^{n}$-invariant open submanifold of $M$. Moreover, we claim that there exists a unique open $\left(\mathbb{C}^{*}\right)^{n}$ orbit in $M$, this orbit and free and is dense in $M$, and it coincides with the free $\left(\mathbb{C}^{*}\right)^{n}$ orbit in $V_{p}$ for each $p$. To see this, we consider the fundamental vector fields $\xi_{1}, \ldots, \xi_{n}$ of the $\left(S^{1}\right)^{n}$-action with respect to the coordinate one-dimensional subtori. We think of them as holomorphic sections $M \rightarrow T^{1,0} M \cong T M$ of the holomorphic tangent bundle $T^{1,0} M$ of $M$. The $n$-th exterior product $\bigwedge^{n} T^{1,0} M \rightarrow M$ is a holomorphic line bundle and $\xi_{1} \wedge \cdots \wedge \xi_{n}$ is a holomorphic section of this line bundle. A point $x \in M$ belongs to an open $\left(\mathbb{C}^{*}\right)^{n}$ orbit if and only if $\left(\xi_{1} \wedge \cdots \wedge \xi_{n}\right)(x)$ is not zero. This means that the union of the open 
$\left(\mathbb{C}^{*}\right)^{n}$ orbits is the complement of the zero locus of a holomorphic section. Since the zero locus is a complex analytic subvariety of $M$ and $M$ is connected, the union of the open $\left(\mathbb{C}^{*}\right)^{n}$ orbits is either empty, or it is open, dense, and connected. The claim then follows from the facts that there exists at least one $V_{p}$, it contains a free and open $\left(\mathbb{C}^{*}\right)^{n}$ orbit, and every two distinct orbits are disjoint.

In particular, $X$ is connected and dense in $M$.

The connected components of the fixed point sets of the circle subgroups of $\left(S^{1}\right)^{n}$ are closed complex submanifolds of $X$. If such a submanifold has complex codimension one, then, in analogy with the toric topology literature, we call it a characteristic submanifold of $X$ (cf. [14, p. 240]).

Since $X$ is a union of finitely many $V_{p}$ s and each $V_{p}$ has only finitely many characteristic submanifolds, there are only finitely many characteristic submanifolds in $X$. Denote them

$$
X_{1}, \ldots, X_{m}
$$

Let $T_{i}$ be the subgroup of $T$ that fixes $X_{i}$. If a compact group acts faithfully on a connected manifold then at every fixed point the linear isotropy representation is faithful. Therefore, the linear isotropy representation of $T_{i}$ at any point $q$ of $X_{i}$ is faithful. Since $T_{i}$ acts holomorphically and fixes $X_{i}$, we get a faithful representation of $T_{i}$ on the one-dimensional complex space $T_{q} X / T_{q} X_{i}$. This gives an injection $T_{i} \rightarrow S^{1}$, where $S^{1}$ acts on $T_{q} X / T_{q} X_{i}$ by scalar multiplication. By continuity, this injection is independent of the choice of point $q$ in $X_{i}$. Since, by assumption, $T_{i}$ contains a circle subgroup of $T$, this injection is an isomorphism. Let

$$
\lambda_{i}: S^{1} \rightarrow T_{i} \subset\left(S^{1}\right)^{n}
$$

be the inverse of this isomorphism, composed with the inclusion map into $\left(S^{1}\right)^{n}$.

We define an abstract simplicial complex:

$$
\Sigma:=\left\{I \subseteq\{1, \ldots, m\} \mid \bigcap_{i \in I} X_{i} \neq \emptyset\right\} .
$$

To each simplex $I \in \Sigma$ we assign the cone

$$
C_{I}:=\operatorname{pos}\left(\lambda_{i} \mid i \in I\right):=\left\{\sum_{i \in I} a_{i} \lambda_{i} \mid a_{i} \geq 0\right\}
$$

in $\operatorname{Lie}\left(S^{1}\right)^{n}$.

Example 9. Take $\mathbb{C}^{n}$ with coordinates $z_{1}, \ldots, z_{n}$. Let $\left(S^{1}\right)^{n}$ act on it with weights $\alpha_{1}, \ldots, \alpha_{n} \in \operatorname{Hom}\left(\left(S^{1}\right)^{n}, S^{1}\right) \subset\left(\operatorname{Lie}\left(S^{1}\right)^{n}\right)^{*}$. Suppose that the action is faithful; then $\alpha_{1}, \ldots, \alpha_{n}$ are a $\mathbb{Z}$-basis of $\operatorname{Hom}\left(\left(S^{1}\right)^{n}, S^{1}\right)$. The characteristic submanifolds are the coordinate hyperplanes $\left\{z_{i}=0\right\}$ for $i=1, \ldots, n$. The homomorphisms $\lambda_{1}, \ldots, \lambda_{n}$ are the basis to $\operatorname{Hom}\left(S^{1},\left(S^{1}\right)^{n}\right) \subset \operatorname{Lie}\left(S^{1}\right)^{n}$ that is dual to $\alpha_{1}, \ldots, \alpha_{n}$.

Recall that a cone in $\operatorname{Lie}\left(S^{1}\right)^{n}$ is unimodular if it is generated by part of a $\mathbb{Z}$-basis of $\operatorname{Hom}\left(S^{1},\left(S^{1}\right)^{n}\right)$.

Returning to our general case -

Lemma 10. The cones $C_{I}$, for $I \in \Sigma$, are unimodular. 
Proof. Let $I \in \Sigma$. By the definition of $\Sigma$, this means that the intersection $\bigcap_{i \in I} X_{i}$ is nonempty. Let $q$ be a point in this intersection. Let $p$ be a fixed point such that $q \in V_{p}$. Since $V_{p}$ is isomorphic to some $\mathbb{C}_{\alpha_{1}} \oplus \cdots \oplus \mathbb{C}_{\alpha_{n}}$ on which the action is faithful, the lemma follows from Example 9.

Fix a point $q$ in the free $\left(\mathbb{C}^{*}\right)^{n}$ orbit in $X$. For any $\xi \in \operatorname{Lie}\left(S^{1}\right)^{n}$, consider the curve

$$
c_{q}^{\xi}: \mathbb{R} \rightarrow X
$$

that is given by

$$
c_{q}^{\xi}(r):=\exp (-r J \xi) \cdot q \quad \text { for } r \in \mathbb{R}
$$

where exp: $\operatorname{Lie}\left(\mathbb{C}^{*}\right)^{n} \rightarrow\left(\mathbb{C}^{*}\right)^{n}$ is the exponential map and where $J$ denotes multiplication by $i$ in $\operatorname{Lie}\left(\mathbb{C}^{*}\right)^{n}$.

Denote by $C_{I}^{0}$ the relative interior of the cone $C_{I}$. Denote

$$
X_{I}=\bigcap_{i \in I} X_{i} \quad \text { and } \quad X_{I}^{0}=\bigcap_{i \in I} X_{i} \backslash \bigcup_{j \notin I} X_{j} .
$$

Lemma 11. Let $\xi \in \operatorname{Lie}\left(S^{1}\right)^{n}$ and $I \in \Sigma$. Then $\xi \in C_{I}^{0}$ if and only if the curve $c_{q}^{\xi}(r)$ converges as $r \rightarrow-\infty$ to a point $q^{\prime}$ in $X_{I}^{0}$. Moreover, in this case the limit point $q^{\prime}$ belongs to $V_{p}$ for every $p$ such that $V_{p} \cap X_{I} \neq \emptyset$.

Proof. Suppose that $\xi \in C_{I}^{0}$. By the definition of $\Sigma, X_{I}$ is nonempty. Let $p$ be such that $V_{p}$ meets $X_{I}$. Without loss of generality assume that $I=\{1, \ldots, k\}$ and that the characteristic submanifolds that meet $V_{p}$ are $X_{1}, \ldots, X_{n}$. Let $\alpha_{1}, \ldots, \alpha_{n}$ denote the isotropy weights at $p$. The assumption that $\xi \in C_{I}^{0}$ exactly means that $\left\langle\xi, \alpha_{i}\right\rangle$ is positive for $i=1, \ldots, k$ and zero for $i=k+1, \ldots, n$. Fix an isomorphism $\left(z_{1}, \ldots, z_{n}\right): V_{p} \rightarrow \mathbb{C}^{n}=\mathbb{C}_{\alpha_{1}} \oplus \ldots \oplus \mathbb{C}_{\alpha_{n}}$ such that $z_{i}(q)=1$ for all $i$. In these coordinates, the curve $c_{q}^{\xi}(r)$ is represented as

$$
\left(z_{1}, \ldots, z_{n}\right)\left(c_{q}(r)\right)=\left(e^{2 \pi r\left\langle\xi, \alpha_{1}\right\rangle}, \ldots, e^{2 \pi r\left\langle\xi, \alpha_{n}\right\rangle}\right) .
$$

As $r$ approaches $-\infty$, the curve in $\mathbb{C}^{n}$ approaches the point $(\underbrace{0, \ldots, 0}_{k}, \underbrace{1, \ldots, 1}_{n-k})$.

On the other hand, the coordinates take each intersection $V_{p} \cap X_{i}$ to the coordinate hyperplane $\left\{\left(z_{1}, \ldots, z_{n}\right) \mid z_{i}=0\right\}$, and they take the intersection $V_{p} \cap X_{I}^{0}$ to the set $\left\{\left(z_{1}, \ldots, z_{n}\right) \mid z_{i}=0\right.$ iff $\left.1 \leq i \leq k\right\}$. So the curve approaches a point in $V_{p} \cap X_{I}^{0}$, as required.

Now suppose that the curve $c_{q}^{\xi}(r)$ converges as $r \rightarrow-\infty$ to a point in $X_{I}^{0}$. Let $p$ be such that this limit point is contained in $V_{p}$. As before, without loss of generality assume that $I=\{1, \ldots, k\}$ and that the characteristic submanifolds that meet $V_{p}$ are exactly $X_{1}, \ldots, X_{n}$; fix an isomorphism $\left(z_{1}, \ldots, z_{n}\right): V_{p} \rightarrow \mathbb{C}^{n}=\mathbb{C}_{\alpha_{1}} \oplus \ldots \oplus \mathbb{C}_{\alpha_{n}}$ such that $z_{i}(q)=1$ for all $i$; the curve $c_{q}^{\xi}(r)$ is represented as $\left(z_{1}, \ldots, z_{n}\right)\left(c_{q}(r)\right)=$ $\left(e^{2 \pi r\left\langle\xi, \alpha_{1}\right\rangle}, \ldots, e^{2 \pi r\left\langle\xi, \alpha_{n}\right\rangle}\right)$. Since the curve approaches a limit as $r \rightarrow-\infty$, the pairings $\left\langle\xi, \alpha_{i}\right\rangle$ are nonnegative for all $i=1, \ldots, n$. Since this limit is in $X_{I}^{0}$, the pairings are positive for every $i \in I$ and they vanish for every $i \in\{1, \ldots, n\} \backslash I$. Thus, $\xi \in C_{I}^{0}$ as required.

Corollary 12. (1) For every $I, J \in \Sigma$, if $I \neq J$, then $C_{I}^{0} \cap C_{J}^{0}=\emptyset$.

(2) For every $I, J \in \Sigma$,

$$
C_{I} \cap C_{J}=C_{I \cap J}
$$


(3) The collection of cones

$$
\Delta:=\left\{C_{I} \mid I \in \Sigma\right\}
$$

is a fan, that is, every face of every cone in $\Delta$ is itself in $\Delta$, and the intersection of every two cones in $\Delta$ is a common face.

Proof. Part (1) follows from Lemma 11 because the sets $X_{I}^{0}$ are disjoint. Part (3) follows from Part (2).

For Part (2), we only need to show the inclusion $C_{I} \cap C_{J} \subseteq C_{I \cap J}$, because the opposite inclusion is trivial. Let $\xi \in C_{I} \cap C_{J}$. Let $I^{\prime} \subset I$ and $J^{\prime} \subset J$ be the subsets such that $\xi \in C_{I^{\prime}}^{0}$ and $\xi \in C_{J^{\prime}}^{0}$. Then $C_{I^{\prime}}^{0} \cap C_{J^{\prime}}^{0} \neq \emptyset$. By Part (1), $I^{\prime}=J^{\prime}$. Let $L=I^{\prime}=J^{\prime}$. Then $L \subset I \cap J$, and $\xi \in C_{L}^{0} \subset C_{I \cap J}$.

Lemma 13. For every $I \in \Sigma$, the set $X_{I}$ is an $\left(S^{1}\right)^{n}$-invariant smooth closed complex submanifold of $X$ of complex codimension $|I|$, it is connected, and it contains a fixed point.

Proof. Fix $I \in \Sigma$.

Since each of the sets $X_{i}$, for $i \in I$, is closed in $X$, so is the intersection $X_{I}$ of these sets.

Since $X$ is the union of open subsets $V_{p}$, and because every intersection $V_{p} \cap X_{I}$ is an $\left(S^{1}\right)^{n}$-invariant complex submanifold of codimension $|I|$ in $V_{p}$, we deduce that $X_{I}$ is itself an $\left(S^{1}\right)^{n}$-invariant complex submanifold of codimension $|I|$ in $X$. It remains to show that $X_{I}$ is connected and contains a fixed point.

Choose any $\xi \in C_{I}^{0}$ (for example, we may take $\xi=\sum_{i \in I} \lambda_{i}$ ), and choose any $q$ in the free $\left(\mathbb{C}^{*}\right)^{n}$ orbit in $X$. By Lemma 11, the curve $c_{q}^{\xi}(r)$ converges as $r \rightarrow-\infty$; let $q^{\prime}$ be its limit. Also by Lemma 11, for every $p$ such that $V_{p} \cap X_{I} \neq \emptyset$, the limit point $q^{\prime}$ belongs to $V_{p}$. Since $X_{I}$ is the union over such $p$ of the subsets $V_{p} \cap X_{I}$, and because each of these subsets is connected and contains $q^{\prime}$, the union $X_{I}$ is connected. Also, every $p$ such that $V_{p} \cap X_{I} \neq \emptyset$ belongs to $V_{p} \cap X_{I}$; because the set of such $p$ s is nonempty, $X_{I}$ contains a fixed point.

Corollary 14. In the fan $\Delta$, every cone is contained in an $n$-dimensional cone.

Proof. Every cone in the fan has the form $C_{I}$ for some $I \in \Sigma$. By Lemma 13, the set $X_{I}$ contains a fixed point; let $p$ be such a fixed point. Since $V_{p}$ was chosen as in Lemma 7 , by Example 9 there exist exactly $n$ characteristic submanifolds, say, $X_{j}$ for $j \in J \subset\{1, \ldots, m\}$ with $|J|=n$, that pass through $p$. Then $J \in \Sigma$, and $C_{J}$ is an $n$-dimensional cone in $\Delta$ that contains $C_{I}$.

\section{Isomorphism of the subset $X$ with a toric manifold}

Let $M$ be a connected complex manifold of complex dimension $n$, let the torus $\left(S^{1}\right)^{n}$ act on $M$ faithfully by biholomorphisms, and assume that this action extends to a holomorphic $\left(\mathbb{C}^{*}\right)^{n}$-action. The set of fixed points is discrete; assume that it is nonempty and finite.

In Section 4 we described an open subset $X$ of $M$ and a unimodular fan $\Delta$. Let $M_{\Delta}$ be the toric variety that is associated to the fan $\Delta$.

Lemma 15. There exists an $\left(S^{1}\right)^{n}$-equivariant biholomorphism between $M_{\Delta}$ and $X$. 
We recall some properties of the set $X$ and the fan $\Delta$. Let $F=M^{\left(S^{1}\right)^{n}}$ denote the fixed point set. For every fixed point $p \in F$, let $\alpha_{p, 1}, \ldots, \alpha_{p, n}$ denote the isotropy weights of the torus action at $p$.

(1) The set $X$ is the union over $p \in F$ of subsets $V_{p}$, such that each $V_{p}$ is an invariant open neighbourhood of $p$ that is equivariantly biholomorphic to the linear representation $\mathbb{C}_{\alpha_{p, 1}} \oplus \cdots \oplus \mathbb{C}_{\alpha_{p, n}}$.

(2) The $n$-dimensional cones in $\Delta$ are in bijection with the fixed point sets $p \in F$, and the cone corresponding to the fixed point $p$ is $\operatorname{pos}\left(\lambda_{i_{1}}, \ldots, \lambda_{i_{n}}\right)$, where $\lambda_{i_{1}}, \ldots, \lambda_{i_{n}}$ is a basis of $\operatorname{Lie}\left(S^{1}\right)^{n}$ that is dual to the basis $\alpha_{p, 1}, \ldots, \alpha_{p, n}$ of $\left(\operatorname{Lie}\left(S^{1}\right)^{n}\right)^{*}$.

The toric variety $M_{\Delta}$ that is associated to the fan $\Delta$ has similar properties: it is the union over $p \in F$ of invariant subsets $V_{p}^{\prime}$, and every $V_{p}^{\prime}$ is equivariantly biholomorphic to $\mathbb{C}_{\alpha_{p, 1}} \oplus \ldots \oplus \mathbb{C}_{\alpha_{p, n}}$.

Lemma 15 follows immediately from these properties of $X$ and $M_{\Delta}$, by the following lemma.

Lemma 16. Let $X$ and $X^{\prime}$ be complex manifolds of complex dimension n, equipped with holomorphic $\left(\mathbb{C}^{*}\right)^{n}$-actions. Suppose that there exist open dense $\left(\mathbb{C}^{*}\right)^{n}$ orbits $\mathcal{O}$ in $X$ and $\mathcal{O}^{\prime}$ in $X^{\prime}$. Suppose that there exist invariant open subsets $V_{p}$ in $X$ and $V_{p}^{\prime}$ in $X^{\prime}$, both indexed by $p \in F$, such that $X$ is the union of the sets $V_{p}$ and $X^{\prime}$ is the union of the sets $V_{p}^{\prime}$, and that for each $p \in F$ there exists an equivariant biholomorphism $\varphi_{p}: V_{p} \rightarrow V_{p}^{\prime}$. Then $X$ is equivariantly biholomorphic to $X^{\prime}$.

Proof. Necessarily, $\mathcal{O}$ is contained in each $V_{p}$ and $\mathcal{O}^{\prime}$ is contained in each $V_{p}^{\prime}$. Fix a point $q$ in $\mathcal{O}$ and a point $q^{\prime}$ in $\mathcal{O}^{\prime}$. After possibly composing each $\varphi_{p}$ by the action of an element of $\left(\mathbb{C}^{*}\right)^{n}$, we may assume that $\varphi_{p}(q)=q^{\prime}$ for each $p \in F$. So, for each $p$ and $\tilde{p} \in F$, the maps $\varphi_{p}$ and $\varphi_{\tilde{p}}$ coincide at the point $q$. By equivariance, $\varphi_{p}$ and $\varphi_{\tilde{p}}$ coincide on all of $\mathcal{O}$; by continuity, they coincide on the entire overlap $V_{p} \cap V_{\tilde{p}}$. Thus, the $\varphi_{p}$ fit together into a map

$$
\varphi=\bigcup_{p} \varphi_{p}: X \rightarrow X^{\prime}
$$

This map is holomorphic, equivariant, and onto. Similarly, the inverses $\psi_{p}:=\varphi_{p}{ }^{-1}$ fit together into a map

$$
\psi=\bigcup_{p} \psi_{p}: X^{\prime} \rightarrow X
$$

We have that $\psi \circ \varphi=\operatorname{id}_{X}$ and $\varphi \circ \psi=\operatorname{id}_{X^{\prime}}$; thus, $\varphi: X \rightarrow X^{\prime}$ is an equivariant biholomorphism, as required.

\section{The compact case}

Let $M$ be a connected complex manifold of complex dimension $n$, with a faithful $\left(S^{1}\right)^{n}$-action, with fixed points.

Suppose that $M$ is compact. In Section 2 we extended the $\left(S^{1}\right)^{n}$-action to a holomorphic $\left(\mathbb{C}^{*}\right)^{n}$-action. In Section 4 we described an open subset $X$ of $M$ and we associated to it a fan $\Delta$.

Lemma 17. The fan $\Delta$ is complete. 
We begin by proving a special case:

Lemma 18. Let $M^{\prime}$ be a complex manifold of complex dimension one, equipped with a faithful holomorphic action of $S^{1}$ with at least one fixed point. Suppose that $M^{\prime}$ is compact and connected. Then $M^{\prime}$ is equivariantly biholomorphic to $\mathbb{C P}^{1}$ with a standard $\mathbb{C}^{*}$-action.

Proof. Consider the $S^{1}$-action on $M^{\prime}$. Near a fixed point, it is isomorphic to the restriction of either the standard $S^{1}$-action on $\mathbb{C}$ or the opposite $S^{1}$-action on $\mathbb{C}$ to an invariant neighbourhood of the origin in $\mathbb{C}$.

Consider the flow that is generated by $-J \xi$, where $\xi$ generates the $S^{1}$-action. If the $S^{1}$-action near a fixed point is standard, then the trajectories of this flow converge to the fixed point as their parameter approaches $-\infty$. If the $S^{1}$-action near a fixed point is opposite from standard, then the trajectories of this flow converge to the fixed point as their parameter approaches $\infty$.

Outside the fixed point set, the action is free. The quotient $M^{\prime} / S^{1}$ is ${ }^{5}$ a real onemanifold with boundary; its boundary is exactly the image of the fixed point set. Since $M^{\prime}$ is compact and connected and contains a fixed point, and by the classification of one-manifolds, the quotient $M^{\prime} / S^{1}$ must be a closed segment.

The flow on $M^{\prime}$ that is generated by $-J \xi$ descends to a flow on the interior of $M^{\prime} / S^{1}$ that does not have fixed points. For each boundary component, the flow approaches that component either as its parameter approaches $\infty$ or as the parameter approaches $-\infty$. Necessarily, it approaches one boundary component when the parameter approaches $\infty$ and it approaches the other boundary component when the parameter approaches $-\infty$.

The corresponding fan must then be equal to the fan of $\mathbb{C P}^{1}$, and the manifold is equivariantly biholomorphic to $\mathbb{C P}^{1}$ by Lemma 16 .

We now return to the setup of Lemma 17: We have a connected complex manifold $M$ of complex dimension $n$, with a faithful $\left(S^{1}\right)^{n}$-action, with fixed points. We assume that $M$ is compact. We consider the open subset $X$ of $M$ and the associated fan $\Delta$ as described in Section 4.

Lemma 19. Every $n-1$ dimensional cone in $\Delta$ is a common face of two $n$-dimensional cones in $\Delta$.

Proof. Let $C_{I}$ be an $n-1$ dimensional cone in $\Delta$, corresponding to the subset $I=$ $\left\{i_{1}, \ldots, i_{n-1}\right\}$ of $\{1, \ldots, m\}$.

Let $T_{I}$ be the codimension one subtorus of $\left(S^{1}\right)^{n}$ that is generated by the circles $T_{i}$ for $i \in I$. By Lemma 13, $X_{I}$ is a connected complex manifold of dimension one, equipped with a faithful holomorphic action of the circle $\left(S^{1}\right)^{n} / T_{I}$ with at least one fixed point. We will now show that $X_{I}$ is compact, and will deduce Lemma 19 from Lemma 18.

First note that $X_{I}$ is a connected component of the fixed point set of $T_{I}$ in $X$. This follows from the facts that $X_{I}$ is connected (by Lemma 13) and that, for each of the subsets $V_{p}$, if the intersection $V_{p} \cap X_{I}$ is nonempty then it is a connected component of the fixed point set of $T_{I}$ in $V_{p}$. Let $N$ denote the connected component of the

\footnotetext{
${ }^{5}$ Here, "is" means that there exists a unique manifold-with-boundary structure on $M^{\prime} / S^{1}$ such that a function is smooth if and only if its pullback to $M^{\prime}$ is smooth.
} 
fixed point set of $T_{I}$ in $M$ that contains $X_{I}$. As in any holomorphic torus action on a complex manifold, $N$ is an $\left(S^{1}\right)^{n}$-invariant closed complex submanifold of $M$. By examining $N$ near a point of $X_{I}$, we deduce that $N$ has complex dimension one. Since $N$ is closed in $M$ and $M$ is compact, $N$ is compact. By Lemma 18, $N$ is equivariantly biholomorphic to $\mathbb{C P}^{1}$ with a standard action of the circle $\left(S^{1}\right)^{n} / T_{I}$. In particular, $N$ contains two fixed points; denote them $p^{\prime}$ and $p^{\prime \prime}$. The intersection $V_{p^{\prime}} \cap N$, being a $\left(\mathbb{C}^{*}\right)^{n}$-invariant neighbourhood of $p^{\prime}$ in $N$, must be all of $N \backslash\left\{p^{\prime \prime}\right\}$. Similarly, the intersection $V_{p^{\prime \prime}} \cap N$ is all of $N \backslash\left\{p^{\prime}\right\}$. So $N$ is contained in the union $X$ of the sets $V_{p}$, and so $N$ must be equal to $X_{I}$. Thus, $X_{I}$ is equivariantly biholomorphic to $\mathbb{C P}^{1}$ with a standard action of the circle $\left(S^{1}\right)^{n} / T_{I}$. This implies the result of Lemma 19.

We are now ready to prove Lemma 17.

Proof of Lemma 1\%. Let $|\Delta|$ denote the union of the cones in $\Delta$, and let $\left|\Delta^{n-2}\right|$ denote the union of the cones in $\Delta$ that have codimension $\geq 2$. The complement $\operatorname{Lie}\left(S^{1}\right)^{n} \backslash$ $\left|\Delta^{n-2}\right|$ is connected, open, and dense in $\operatorname{Lie}\left(S^{1}\right)^{n}$.

By Lemma 19, the union of the relative interiors of the faces of $\Delta$ of dimension $(n-1)$ and of dimension $n$ is open in $\operatorname{Lie}\left(S^{1}\right)^{n}$. This union is $|\Delta| \backslash\left|\Delta^{n-2}\right|$. Thus, $|\Delta| \backslash\left|\Delta^{n-2}\right|$ is also open in $\operatorname{Lie}\left(S^{1}\right)^{n} \backslash\left|\Delta^{n-2}\right|$.

However, because $|\Delta|$ is closed in $\operatorname{Lie}\left(S^{1}\right)^{n}$, we also have that $|\Delta| \backslash\left|\Delta^{n-2}\right|$ is closed in $\operatorname{Lie}\left(S^{1}\right)^{n} \backslash\left|\Delta^{n-2}\right|$.

Since $|\Delta| \backslash\left|\Delta^{n-2}\right|$ is open and closed in $\operatorname{Lie}\left(S^{1}\right)^{n} \backslash\left|\Delta^{n-2}\right|$ and $\operatorname{Lie}\left(S^{1}\right)^{n} \backslash\left|\Delta^{n-2}\right|$ is connected, we deduce that $|\Delta| \backslash\left|\Delta^{n-2}\right|$ is either empty or is equal to all of $\operatorname{Lie}\left(S^{1}\right)^{n} \backslash$ $\left|\Delta^{n-2}\right|$.

As, by assumption, $M$ has a fixed point, $\Delta$ has at least one $n$-dimensional cone, so $|\Delta| \backslash\left|\Delta^{n-2}\right|$ is not empty. So $|\Delta| \backslash\left|\Delta^{n-2}\right|$ is equal to all of $\operatorname{Lie}\left(S^{1}\right)^{n} \backslash\left|\Delta^{n-2}\right|$. Taking the closures, we deduce that $|\Delta|=\operatorname{Lie}\left(S^{1}\right)^{n}$, as required.

We are now ready to prove our main theorem.

Proof of Theorem 1. Lemma 16 gives an equivariant biholomorphism

$$
\varphi: M_{\Delta} \rightarrow X .
$$

By Lemma 17 , the fan $\Delta$ is complete. This implies that the toric variety $M_{\Delta}$ is compact. So $X$ must be compact. Since $M$ is Hausdorff and connected, and $X$ is a subset that is both compact and open, $X$ is all of $M$. So $\varphi$ defines an equivariant biholomorphism from $M_{\Delta}$ to $M$, as required.

\section{Acknowledgments}

We would like to thank the anonymous referee for helpful suggestions. Yael Karshon would also like to thank Ignasi Mundet i Riera and Jaume Amorós for helpful discussions. Hiroaki Ishida was supported by JSPS Research Fellowships for Young Scientists. This work is partially supported by the JSPS Institutional Programme for Young Researcher Overseas Visits "Promoting international young researchers in mathematics and mathematical sciences led by OCAMI". Yael Karshon is partially supported by the Natural Sciences and Engineering Research Council of Canada. 


\section{References}

[1] M. Audin, Torus actions on symplectic manifolds, Progress in Mathematics 93 Birkhäuser Verlag, Basel, 2nd revised edn., 2004.

[2] V.M. Buchstaber and T.E. Panov, Torus actions and their applications in topology and combinatorics, University Lecture series, Amer. Math. Soc., Providence, RI, 24, 2002.

[3] D.A. Cox, J.B. Little, and H.K. Schenck, Toric varieties, Graduate Studies in Mathematics 124 Amer. Math. Soc., 2011.

[4] M.W. Davis and T. Januszkiewicz, Convex polytopes, Coxeter orbifolds, and torus actions, Duke Math. J. 62 (1991), 417-451.

[5] T. Delzant, Hamiltoniens périodiques et image convexe de l'application moment, Bull. Soc. Math. France 116 (1988), 315-319.

[6] M. Grossberg and Y. Karshon, Equivariant index and the moment map for completely integrable torus actions, Adv. Math. 133 (1998), 185-223.

[7] V. Guillemin and S. Sternberg, Convexity properties of the moment mapping, Invent. Math 67 (1982), 491-513.

[8] - Geometric quantization and multiplicities of group representations, Invent. Math 67 (1982), 515-538.

[9] H. Ishida and M. Masuda, Todd genera of complex torus manifolds, Algebr. Geom. Topol. 12 (2012) 1777-1788.

[10] Y. Karshon, Hamiltonian actions of Lie groups, PhD thesis, Harvard University, 1993.

[11] G. Kempf, F.F. Knutson, D. Mumford, and B. Saint-Donat, Toroidal Embeddings I, Lecture Notes in Math., 339 Springer, Berlin, 1973.

[12] S. Kobayashi and K. Nomizu, Foundation of differential geometry Vol. II, New York-LondonSydney, Interscience Publishers, John Willey \& Sons, Inc., 1969.

[13] A.A. Kustarev, Equivariant almost complex structures on quasitoric manifolds, Proc. Steklov Inst. Math. 266(1) (2009), 133-141.

[14] M. Masuda, Unitary toric manifolds, multi-fans and equivariant index, Tohoku Math. J. 51 (1999), 237-265.

[15] K. Miyake and T. Oda, Almost homogeneous algebraic varieties under algebraic torus action, in A. Hattori, editor, in: Manifolds - Tokyo 1973 (Proc. Int. Conf. Tokyo, 1973), 373-381, University Tokyo Press, Tokyo, 1975.

[16] H. Sumihiro, Equivariant completion, J. Math. Kyoto University 14(1) (1974), 1-28.

Osaka City University Advanced Mathematical Institute, 3-3-138, Sumiyoshi-ku, Osaka 558-8585, JAPAN

E-mail address: ishida@sci.osaka-cu.ac.jp

Current address: Research Institute for Mathematical Science, Kyoto University, Kyoto 605-8502, JAPAN

E-mail address: ishida@kurims.kyoto-u.ac.jp

Department of Mathematics, University of Toronto, 40 St.George Street, Toronto, ONTARIO M5S 2E4, CANADA

E-mail address: karshon@math.toronto.edu 
